\title{
Measurement of $\operatorname{Br}\left(K_{S} \rightarrow \pi^{+} \pi^{-} \pi^{0}\right)$ and limit on the CP violating decay $\operatorname{Br}\left(K_{S} \rightarrow 3 \pi^{0}\right)$
}

\section{Martin Wache ${ }^{* \dagger}$}

Institut für Physik, Univeristät Mainz, Germany

E-mail: wache@uni-mainz.de

The NA48/1 Collaboration has measured the amplitude of the CP-conserving component of the decay $K_{S}^{0} \rightarrow \pi^{+} \pi^{-} \pi^{0}$ relative to $K_{L}^{0} \rightarrow \pi^{+} \pi^{-} \pi^{0}$ using data taken in 2002. The amplitude has been calculated by measuring the parameter $\lambda$ which describes the interference between the $K_{L}$ and $K_{S}$ decays. From a fit to 19 million reconstructed $K^{0} / \bar{K}^{0} \rightarrow \pi^{+} \pi^{-} \pi^{0}$ events the values $\operatorname{Re}(\lambda)=0.038 \pm 0.010$ and $\operatorname{Im}(\lambda)=-0.013 \pm 0.007$ have been obtained. This corresponds to a branching ratio of $\operatorname{Br}\left(K_{S} \rightarrow \pi^{+} \pi^{-} \pi^{0}\right)=\left(4.7_{-1.7}^{+2.2}(\text { stat })_{-1.5}^{+1.7}(\right.$ syst $\left.)\right) \times 10^{-7}$.

We also searched for the CP violating decay $K_{S} \rightarrow 3 \pi^{0}$ using data taken in the year 2000 . The CP violating amplitude $\eta_{000}=A\left(K_{S} \rightarrow 3 \pi^{0}\right) / A\left(K_{L} \rightarrow 3 \pi^{0}\right)$ has been measured by fitting the lifetime distribution of about 4.9 million reconstructed $K^{0} / \bar{K}^{0} \rightarrow 3 \pi^{0}$ decays. This results in an upper limit on the branching fraction of $\operatorname{BR}\left(K_{S} \rightarrow 3 \pi^{0}\right)<4.7 \times 10^{-7}$ at $90 \%$ confidence level.

International Europhysics Conference on High Energy Physics

July 21 st - 27th 2005

Lisboa, Portugal

\footnotetext{
* Speaker.

†n behalf of the NA48/1 collaboration: Cambridge, Chicago, CERN, Dubna, Edinburgh, Ferrara, Firenze, Mainz, Northwestern, Perugia, Pisa, Saclay, Siegen, Torino, Warsaw, Vienna
} 


\section{Experimental Setup}

The NA48 experiment is located in the North Area of the CERN Super Proton Synchrotron in Geneva, it has been designed to measure direct $\mathrm{CP}$ violation in neutral kaon decays. For this purpose two simultaneous, almost collinear beams have been used, both derived from a proton beam delivered to two fixed targets by the CERN SPS. The far target is located $126 \mathrm{~m}$ before the beginning of the $96 \mathrm{~m}$ long decay region. The near target is located $7.2 \mathrm{~cm}$ above the far beam and $6 \mathrm{~m}$ before the decay region. During the data taking in 2002, the far target had been removed and only the near target has been used to produce a high intensity $K^{0}$ beam.

The main NA48 detector components are the magnetic spectrometer which consist of four drift chambers and a dipole magnet and the liquid krypton electromagnetic calorimeter. The four drift chambers with four views each have a spatial resolution of $120 \mu \mathrm{m}$ per view, resulting in a momentum resolution of $\sigma(p) / p=(0.48 \oplus 0.015 p) \%$. During the data taking in the year 2000 the drift chambers were removed. The electromagnetic calorimeter is 27 radiation length long and segmented transversally in 13212 cells of $2 \times 2 \mathrm{~cm}^{2}$, with spatial and time resolution better than $1.3 \mathrm{~mm}$ and $300 \mathrm{ps}$ respectively. The energy resolution was $\sigma(E) / E=(9 / E \oplus 3.2 / \sqrt{E} \oplus 0.42) \%$. A more detailed description of the detector can be found in [3].

2. $K^{0} \rightarrow 3 \pi^{0}$

For the search of the CP-violating decay $K_{S} \rightarrow 3 \pi^{0}$ data taken in the year 2000 were used. The NA48 run in the year 2000 was split in two periods, a 30-day run with only the far-target in operation and a 40-day run with a high intensity beam from the near-target. During both periods the drift chambers were not in place and the trigger decision was mainly based on a projective readout of the liquid krypton calorimeter. It required a minimum of at least $50 \mathrm{GeV}$ deposited energy, a radius of the energy center-of-gravity less than $15 \mathrm{~cm}$ from the detector axis and a proper lifetime measured from the final collimator less than $9 K_{S}$ lifetimes.

For the event selection the first step was to select good clusters. The cluster energy of good clusters had to be in the range of linear energy response of the calorimeter and the position inside of the fiducal region of the calorimeter. On all combinations of 6 good clusters further selection criteria were applied: all 6 clusters had to be in time and the radial distance of the center-of-gravity at the calorimeter had to lie within the beam spot. The longitudinal vertex along the $\mathrm{z}$ direction was then calculated using the positions and the energies of the clusters in the

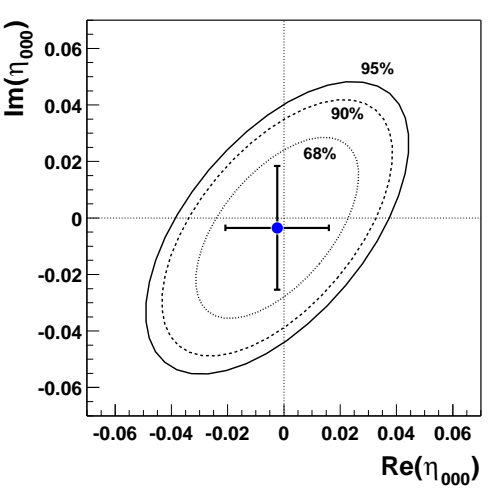

Figure 1: Fit result for $\eta_{000}$, the errors include both statistical and systematic uncertainties. 
liquid krypton calorimeter:

$$
z_{\text {Vertex }}=z_{L k r}-\frac{1}{m_{K}} \sqrt{\sum_{i=1}^{6} \sum_{j=i+1}^{6} E_{i} E_{j}\left[\left(x_{i}-x_{j}\right)^{2}+\left(y_{i}-y_{j}\right)^{2}\right]}
$$

with the longitudinal position $z_{L k r}$ of calorimeter and the nominal kaon mass $m_{K}$. Using the longitudinal vertex position, the invariant two-photon masses were calculated for each of the 15 possible combinations. For each combination a $\chi^{2}$-like value of the two-photon masses was calculated, the combination with the smallest $\chi_{3 \pi^{0}}^{2}$ was accepted if it was less than 90 .

With these selection criteria about $4.9 \times 10^{6} K_{L, S} \rightarrow$

\begin{tabular}{l|c|c} 
& $\operatorname{Re} \eta_{000}$ & $\operatorname{Im} \eta_{000}$ \\
\hline rec. effic. & \pm 0.009 & \pm 0.013 \\
Z resolution & \pm 0.010 & \pm 0.000 \\
beam geom. & \pm 0.005 & \pm 0.007 \\
background & \pm 0.002 & \pm 0.009
\end{tabular}
$3 \pi^{0}$ events were found in the near-target run and about $109 \times 10^{6} K_{L} \rightarrow 3 \pi^{0}$ events in the far-target run data.

At the NA48 targets, $K_{L}$ and $K_{S}$ mesons were produced in equal amounts, the $K \rightarrow 3 \pi^{0}$ intensity as a func-

Table 1: The largest systematic uncertainties for $\eta_{000}$

$$
\begin{aligned}
I_{3 \pi^{0}} \propto & e^{-\Gamma_{L} t}+2 D(p)\left[\operatorname{Re}\left(\eta_{000}\right) \cos \Delta m t\right. \\
& \left.\quad-\operatorname{Im}\left(\eta_{000}\right) \sin \Delta m t\right] e^{-\frac{1}{2}\left(\Gamma_{L}+\Gamma_{S}\right) t}+O\left(\eta_{000}{ }^{2}\right),
\end{aligned}
$$

with the dilution $D(p)=\left(N_{K^{0}}-N_{\bar{K}^{0}}\right) /\left(N_{K^{0}}+N_{\bar{K}^{0}}\right)$, the total $K_{L}$ and $K_{S}$ widths $\Gamma_{L}$ and $\Gamma_{S}$ and the difference $\Delta m$ of the masses of $K_{L}$ and $K_{S}$. To extract the $K_{L} K_{S}$-interference the near-run data was divided by the far-run data applying an geometrical acceptance correction determined with a Monte Carlo simulation: $f_{3 \pi^{0}}(t)=\frac{N_{3 \pi^{0}}^{\text {near }}(t)}{N_{K_{L} \rightarrow 3 \pi^{0}}^{\text {far }}(t)} \frac{\varepsilon_{K_{L} \rightarrow 3 \pi^{0}}^{\text {far }}(t)}{\varepsilon_{K_{L} \rightarrow 3 \pi^{0}}^{\text {near }}(t)}$.

To this ratio a fit was performed in 25 bins of the energy using a linear fit to NA31 data [4] for the dilution. The fit and a detailed study of systematic effects yields the result:

$$
\begin{aligned}
& \operatorname{Re} \eta_{000}=-0.002 \pm 0.011_{\text {stat }} \pm 0.015_{\text {sys }} \\
& \operatorname{Im} \eta_{000}=-0.003 \pm 0.013_{\text {stat }} \pm 0.017_{\text {sys }}
\end{aligned}
$$

with a statistical correlation of $\rho=0.77$ (Fig. 1). Using the branching ratio of $K_{L} \rightarrow 3 \pi^{0}$ and the lifetimes of $K_{L}$ and $K_{S}$, this result turns into an upper limit on the branching fraction of $\mathrm{BR}\left(K_{S} \rightarrow\right.$ $\left.3 \pi^{0}\right)<7.4 \times 10^{-7}$ at $90 \%$ confidence level. The most important sources of uncertainties are listed in Table 1. One can also use the result to improve the knowledge of $\operatorname{Re}(\varepsilon)$ and the CPT violating quantity $\operatorname{Im}(\delta)$ via the Bell-Steinberger relation:

$$
\begin{aligned}
\operatorname{Re}(\delta) & =(-0.2 \pm 2.0) \times 10^{-5} \\
\operatorname{Im}(\varepsilon) & =(166.4 \pm 1.0) \times 10^{-5}
\end{aligned}
$$

A more detailed description of the analysis can be found in [1].

3. $K_{S} \rightarrow \pi^{+} \pi^{-} \pi^{0}$

The data used for the analysis of $K_{S} \rightarrow \pi^{+} \pi^{-} \pi^{0}$ was taken in a 89 day high-intensity neartarget run in 2002. In this data taking period the trigger rejected all events which passed a $K_{S} \rightarrow$ 
$\pi^{+} \pi^{-}$or $\Lambda^{0} \rightarrow p \pi^{-}$hypothesis, and required a ratio of the momenta of the two charged tracks of $P_{\text {larger }} / P_{\text {smaller }}<3.5$ and more than $30 \mathrm{GeV}$ energy deposited in the calorimeters. On top of this the offline level-3 trigger required that two clusters in the electromagnetic calorimeter passed the hypotheses of a $\pi^{0}$ decaying into two gammas at the charged vertex.

In the analysis events were selected with two tracks of opposite charge, coming from the same vertex, and at least two good electromagnetic clusters. If the event had more than two good clusters, the two clusters yielding the best $\pi^{0}$ mass were used. The mass reconstructed for the $\pi^{0}$ had to be inside of a $\pm 7.8 \mathrm{MeV}$ interval and for the kaon $\pm 10.5 \mathrm{MeV}$ interval around the world averages of the corresponding masses (Fig. 2). Using this selection 19 million events were found.

In the decay $K^{0} \rightarrow \pi^{+} \pi^{-} \pi^{0}$ angular momentum eigenvalues other than $l=0$ are allowed. Since the CP conserving decay amplitude of the decay $K_{S} \rightarrow$ $\pi^{+} \pi^{-} \pi^{0}$ with $l=1$ is antisymmetric in the dalitz variable $X=\frac{S_{\pi^{-}}-S_{\pi^{+}}}{m_{\pi^{ \pm}}^{2}}$ while the dominant $K_{L} \rightarrow \pi^{+} \pi^{-} \pi^{0}$

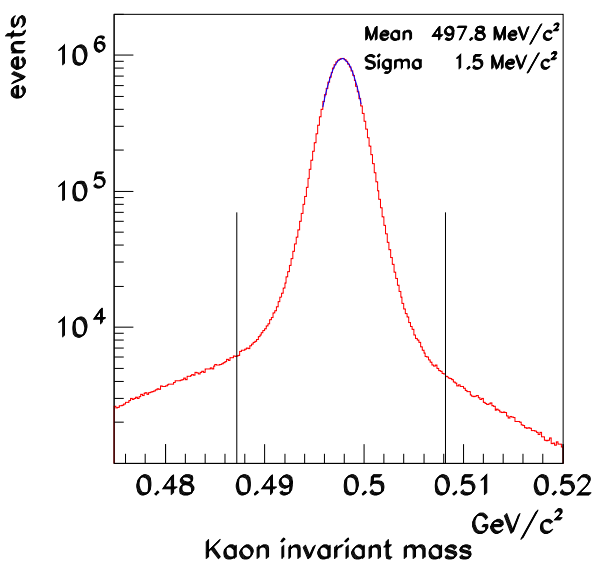

Figure 2: The $K^{0}$ invariant mass. The vertical lines indicate the $K^{0}$ mass cut used in the analysis. $(l=0)$ is symmetric in $X$, one can extract the interference by splitting the data into two samples with $X>0$ and $X<0$. Using the dalitz variable $X$ the intensity of $K^{0} \rightarrow \pi^{+} \pi^{-} \pi^{0}$ events in the NA48-detector can be written as:

$$
I_{\pi^{+} \pi^{-} \pi^{0}}^{X \gtrless 0} e^{-\Gamma_{L} t}+D(p)\left(\operatorname{Re}\left(\eta_{+-0} \pm \lambda\right) \cos \Delta m t-\operatorname{Im}\left(\eta_{+-0} \pm \lambda\right) \sin \Delta m t\right) e^{-\frac{1}{2}\left(\Gamma_{L}+\Gamma_{S}\right) t}+O\left(\eta_{+-0}{ }^{2}, \lambda^{2}\right)
$$

The ratio $V(t)=I_{\pi^{+} \pi^{-} \pi^{0}}^{X>0}(t)-I_{\pi^{+} \pi^{-} \pi^{0}}^{X<0}(t) / I_{\pi^{+} \pi^{-} \pi^{0}}^{X>0}(t)+I_{\pi^{+} \pi^{-} \pi^{0}}^{X<0}(t)$ has been used to extract the interference between $K_{L} \rightarrow \pi^{+} \pi^{-} \pi^{0}$ with $l=0$ and $K_{S} \rightarrow \pi^{+} \pi^{-} \pi^{0}$ with $l=1$. A fit to this ratio was performed in eight bins of the energy using the dilution measured by NA31 [4]. Together with a detailed study of systematic uncertainties we obtained from the fit:

$$
\begin{aligned}
& \operatorname{Re} \lambda=+0.038 \pm 0.008_{\text {stat }} \pm 0.006_{\text {sys }} \\
& \operatorname{Im} \lambda=-0.013 \pm 0.005_{\text {stat }} \pm 0.004_{\text {sys }}
\end{aligned}
$$

with a correlation of $\rho=0.66$. The largest contributions to the systematic uncertainty were from the differences between the different spectrometer magnet polarisation and the knowledge of the dilution. This result for $\lambda$ can be used to calculate a branching ratio of $\operatorname{Br}\left(K_{S} \rightarrow \pi^{+} \pi^{-} \pi^{0}\right)=$ $\left(4.7_{-1.7}^{+2.2}(\text { stat })_{-1.5}^{+1.7}(\right.$ syst $\left.)\right) \times 10^{-7}$. A detailed description of this analysis can be found in [2].

\section{References}

[1] J.R.Batley et al., Phys. Lett. B 610 3-4 (2005) 165-176.

[2] J.R.Batley et al., Phys. Lett. B 630 1-2 (2005) 31-39.

[3] A.Lai et al., Eur. Phys. J. C 22 (2001) 231-254, and

J.R. Batley at al., Phys. Lett B 576 1-2 (2003) 43-54.

[4] R. Carosi et al. (NA31 Collaboration), Phys. Lett. B 237 (1990), 303 